\title{
CONDITIONAL LIMIT THEOREMS FOR THE TERMS OF A RANDOM WALK REVISITED
}

\author{
SHAUL K. BAR-LEV, ${ }^{*}$ University of Haifa \\ ERNST SCHULTE-GEERS, ** Federal Office for Information Security \\ WOLFGANG STADJE, ${ }^{* * *}$ University of Osnabrück
}

\begin{abstract}
In this paper we derive limit theorems for the conditional distribution of $X_{1}$ given $S_{n}=s_{n}$ as $n \rightarrow \infty$, where the $X_{i}$ are independent and identically distributed (i.i.d.) random variables, $S_{n}=X_{1}+\cdots+X_{n}$, and $s_{n} / n$ converges or $s_{n} \equiv s$ is constant. We obtain convergence in total variation of $\mathbb{P}_{X_{1} \mid S_{n} / n=s}$ to a distribution associated to that of $X_{1}$ and of $\mathbb{P}_{n X_{1} \mid S_{n}=s}$ to a gamma distribution. The case of stable distributions (to which the method of associated distributions cannot be applied) is studied in detail.
\end{abstract}

Keywords: Conditional limit theorem; sums of i.i.d. random variables; renewal theory; convergence in total variation; stable distribution

2010 Mathematics Subject Classification: Primary 60F05

Secondary 60K05

\section{Introduction}

In this paper we present some extensions, supplements, and variations of results on the relationship between the sum $S_{n}=X_{1}+\cdots+X_{n}$ of $n$ independent and identically distributed (i.i.d.) random variables and the individual terms $X_{i}$. Formulated in the language of renewal theory, given that the $n$th renewal takes place at time $S_{n}=s_{n}$, what is the asymptotic behavior of the conditional interarrival time distribution $\mathbb{P}_{X_{1} \mid S_{n}=s_{n}}$ for various types of real sequences $s_{n}$ ? This question has been of interest in renewal theory since it was discovered that, for a Poisson process, the first $n$ interarrival times, conditioned on the $n$th arrival taking place at time $s$, have the same distribution as the spacings of an independent sample of $n-1$ uniform random variables on $(0, s)$. In the renewal context the $X_{i}$ will be nonnegative, but for most of our derivations this property is not required.

One new feature of our results is that the mode of convergence is always convergence in total variation. We consider different types of behavior of the sum.

Case A: let $s_{n} / n \rightarrow m \in \mathbb{R}$. We show that in this case $\mathbb{P}_{X_{1} \mid S_{n}=s_{n}}$ converges to the associated distribution with density $\mathrm{e}^{-\zeta x} / \ell(\zeta)$ with respect to $\mathbb{P}_{X_{1}}$, where $\ell(\zeta)$ is the (in general, two-sided) Laplace-Stieltjes transform (LST) of $\mathbb{P}_{X_{1}}$ and $\zeta=\zeta(m) \in \mathbb{R}$ has to be chosen such that $\ell^{\prime}(\zeta) / \ell(\zeta)=-m$. Actually, under standard conditions, the densities converge

\footnotetext{
Received 7 November 2011; revision received 29 November 2012.

* Postal address: Department of Statistics, University of Haifa, Haifa 31905, Israel.

Email address: barlev@stat.haifa.ac.il

Supported by a Mercator professorship of the Deutsche Forschungsgemeinschaft at the University of Osnabrück.

** Postal address: Federal Office for Information Security, Godesberger Allee 185-189, 53175 Bonn, Germany.

Email address: ernst.schulte-geers@bsi.bund.de

*** Postal address: Institute of Mathematics, University of Osnabrück, 49069 Osnabrück, Germany.

Email address: wstadje@uos.de
} 
uniformly on compact sets. Hence, knowledge of the behavior of the sum being distorted results in a change of the underlying distribution of the $X_{i}$, which is achieved by means of multiplication with a certain density (with respect to $\mathbb{P}_{X_{1}}$ ). Our result includes the nondistorted case $m=\mathbb{E}\left[X_{1}\right]$ (note that $\zeta\left(\mathbb{E}\left[X_{1}\right]\right)=0$ ).

Case B: let $s_{n}=s=$ constant and let $X_{1}$ be positive. In this case the summands must get small for large $n$; clearly, $\mathbb{E}\left[n X_{1} \mid S_{n}=s\right]=s$. We introduce $n$ as a scaling factor and prove that (under certain conditions) the density of $\mathbb{P}_{n X_{1} \mid S_{n}=s}$ tends to a gamma density as $n \rightarrow \infty$, with scale and shape parameters depending on $s$ and the behavior of $\mathbb{P}_{X_{1}}$ at 0 (of course, its mean is $s$ ).

For case A, we do not have to assume that the $X_{i}$ are nonnegative. We remark that in case A the function $\ell^{\prime}(\zeta) / \ell(\zeta)$ is increasing so that $m \mapsto \zeta(m)$ is decreasing and we have $\zeta(m)<0$ if $m>\mathbb{E}\left[X_{1}\right]$ and $\zeta(m)>0$ if $m<\mathbb{E}\left[X_{1}\right]$. Thus, the asymptotic density of $\mathbb{P}_{X_{1} \mid S_{n}=s_{n}}$ with respect to $\mathbb{P}_{X_{1}}$, i.e. $\mathrm{e}^{-\zeta x} / \ell(\zeta)$, is decreasing if $m<\mathbb{E}\left[X_{1}\right]$ and increasing if $m>\mathbb{E}\left[X_{1}\right]$. Therefore, the asymptotic distribution is stochastically smaller than $\mathbb{P}_{X_{1}}$ if $m<\mathbb{E}\left[X_{1}\right]$ and stochastically larger if $m>\mathbb{E}\left[X_{1}\right]$.

The reason that the associated (also called conjugate) distribution $Q_{\zeta}$ (the probability measure with density $\mathrm{e}^{-\zeta x} / \ell(\zeta)$ with respect to $\mathbb{P}_{X_{1}}$ ) appears is that the distribution of $X_{1}$ given $S_{n}$ remains the same if the distribution of every $X_{i}$ is changed to $Q_{\zeta}$. Then $\zeta$ is chosen so as to get the 'right' expectation, in the same way as, for example, in the standard asymptotic calculation of probabilities of large deviations. In fact, conditioning on $S_{n}=s_{n}$ with $s_{n} / n$ having a limit different from $\mathbb{E} X_{1}$, implies that large deviations are considered.

The stable distributions do not satisfy the conditions that are required for our results. However, we will show that they can be analyzed directly. Consider first the extreme stable distribution $G_{\alpha}$ with index $\alpha \in(0,2)$, i.e. having characteristic function

$$
\phi_{\alpha}(u)= \begin{cases}\exp \left\{-|u|^{\alpha} \exp \left(-\frac{\mathrm{i} \pi}{2} K(\alpha) \operatorname{sgn} u\right)\right\}, & \alpha \neq 1, \\ \exp \left\{-|u|\left(\frac{\pi}{2}+\mathrm{i}(\operatorname{sgn} u) \log |u|\right)\right\}, & \alpha=1,\end{cases}
$$

where $K(\alpha)=\alpha-1+\operatorname{sgn}(1-\alpha)$. Let $g_{\alpha}(x)$ and $g_{\alpha}^{(n)}(x \mid s)$ be the densities of $G_{\alpha}$ and of $\mathbb{P}_{X_{1} \mid S_{n} / n=s}$, respectively.

- For $\alpha \in(0,1)$, the support of $G_{\alpha}$ is $[0, \infty)$ and we show that

$$
\lim _{n \rightarrow \infty} g_{\alpha}^{(n)}(x \mid s)=\exp \left\{\left(\frac{s}{\alpha}\right)^{\alpha /(1-\alpha)}-\left(\frac{s}{\alpha}\right)^{1 /(\alpha-1)} x\right\} g_{\alpha}(x) \text { for all } s>0, x>0 .
$$

- For $\alpha=1$, the support of $G_{\alpha}$ is $\mathbb{R}$ and we show that

$$
\lim _{n \rightarrow \infty} g_{1}^{(n)}(x \mid s)=\exp \left\{\mathrm{e}^{-s-1}(1+s-x)\right\} g_{1}(x) \quad \text { for all } s \in \mathbb{R}, x \in \mathbb{R} .
$$

- For $\alpha \in(1,2)$, the support of $G_{\alpha}$ is $\mathbb{R}$ and

$$
\begin{aligned}
\lim _{n \rightarrow \infty} g_{\alpha}^{(n)}(x \mid s) & \\
= & \begin{cases}\exp \left\{-\left(\frac{|s|}{\alpha}\right)^{\alpha /(1-\alpha)}-\left(\frac{|s|}{\alpha}\right)^{1 /(\alpha-1)} x\right\} g_{\alpha}(x) & \text { for all } s<0, x \in \mathbb{R}, \\
g_{\alpha}(x) & \text { for all } s \geq 0, x \in \mathbb{R} .\end{cases}
\end{aligned}
$$


For the nonextreme stable distributions, we prove that the conditional densities converge pointwise to the unconditional density.

Weak convergence of $\mathbb{P}_{X_{1} \mid S_{n}=s_{n}}$ to the appropriate associated distribution as in case B was derived in [15] under more restrictive conditions (the motivation there is from statistical mechanics). The proof in [15] was based on an unpublished result in the dissertation of Zabell [17]. We sharpen Theorem 2 of [15] and establish convergence in total variation. In fact, we obtain, for example, even uniform convergence of the density of $\mathbb{P}_{X_{1} \mid S_{n}=n m}$ on compact sets to the limiting density if $X_{1}$ has a bounded density. Our result in case B is related, though not directly comparable, to that of [4] on 'thickened renewal processes'. In [4] the weak limits of the distributions $\mathbb{P}_{n X_{1} \mid S_{n} \leq s}$ and $\mathbb{P}_{n X_{1} \mid S_{n} \leq s<S_{n+1}}$ are studied, which are not quite the same as $\mathbb{P}_{n X_{1} \mid S_{n}=s}$, for which we establish convergence in total variation. The methods are also different (renewal-theoretic versus complex-analytic).

The vast literature on conditional limit theorems for random walks, except for [15] and the older references from statistical physics cited therein, deals with conditions that are of another type than $S_{n}=s$; see, e.g. [3], [6], [9], and [12]. Asymptotic results for linear combinations of $X_{1}, \ldots, X_{n}$ given $S_{n}$ can be found in [7], [8], [10], and [13]. A functional limit theorem under the condition $S_{n}=$ constant is given in [14], in [18] a convergence result for expectations conditioned on a sum is proved, and in [2] an approximation for the distribution of $\left(X_{1}, \ldots, X_{k}\right)$ given $S_{n}$ for $n \rightarrow \infty$ and $k / n \rightarrow 0$ is developed. The asymptotics in the case of stable distributions have not been studied before.

The editor points out that case A of the present paper is related to discussions in [11], where the relevant results as referred to as 'Boltzmann's law'; see also [1, Chapter VI].

Our motivation stems from inventory theory where the $X_{i}$ represent individual demands for different storage sites and their sum is of course the pooled total demand. Then, given the total demand, what can be said about an individual demand? In other words, given $S_{n} / n=s$, what is the distribution of $X_{i}$ ? Suppose that the total demand $S_{n}$ for $n$ inventories becomes known but not the way the demand is split among the individual storage places. Then on average every inventory will have to satisfy the same amount on demand, namely $S_{n}$. Our results provide information on the conditional distribution of the individual demands. For background on pooling of stochastic demands, see [16].

The paper is organized as follows. In Sections 2 and 4 we derive the total variation limit theorems for the conditional distribution of $X_{1}$, given that $S_{n}=s_{n}$, for certain real sequences, announced in cases A-B. In between in Section 3 the asymptotic behavior of $\mathbb{P}_{X_{1} \mid S_{n}=n s}$ for the stable distributions is determined.

\section{Conditional limit theorems for $s_{n} / n \rightarrow m$}

We now study the asymptotic behavior of $\mathbb{P}_{X_{1} \mid S_{n}=s_{n}}$ for real sequences $s_{n}$ satisfying $\mid n^{-1} s_{n}-$ $m \mid=O\left(n^{-1 / 2}\right)$ for some $m \in \mathbb{R}$ and make the following assumptions.

(A1) The set $\{\zeta \in \mathbb{R} \mid \ell(\zeta)<\infty\}$ contains a nonempty open interval $U$.

(A2) There is a $\zeta=\zeta(m) \in U$ for which $\ell^{\prime}(\zeta) / \ell(\zeta)=-m$.

(A3) $X_{1}$ has a density $f$.

(A4) For $\zeta=\zeta(m)$, the characteristic function $\phi_{\zeta}$ of the associated distribution with Lebesgue density e $\mathrm{e}^{-\zeta x} f(x) / \ell(\zeta)$, say $Q_{\zeta}$, satisfies $\int\left|\phi_{\zeta}(t)\right|^{n_{0}} \mathrm{~d} t<\infty$ for some $n_{0} \in \mathbb{N}$. 
Note that the function $-\ell^{\prime} / \ell: U \rightarrow \mathbb{R}$ is one-to-one, so if $m$ is in its range, $\zeta(m)$ is uniquely determined, and $Q_{\zeta}$ has expected value $m$.

Theorem 1. If (A1)-(A4) hold then, for every compact set $K \subset \mathbb{R}$,

$$
\lim _{n \rightarrow \infty} \sup _{x \in K}\left|\frac{f_{n-1}\left(s_{n}-x\right)}{f_{n}\left(s_{n}\right)}-\frac{\mathrm{e}^{-\zeta x}}{\ell(\zeta)}\right|=0 .
$$

In particular, the Lebesgue density $f(x) f_{n-1}\left(s_{n}-x\right) / f_{n}\left(s_{n}\right)$ of $\mathbb{P}_{X_{1} \mid S_{n}=s_{n}}$ converges pointwise to that of $Q_{\zeta}$, so the standard version of $\mathbb{P}_{X_{1} \mid S_{n}=s_{n}}$ converges to $Q_{\zeta}$ in total variation. If $f$ is bounded, the density of $\mathbb{P}_{X_{1} \mid S_{n}=s_{n}}$ converges uniformly on compact sets.

Proof. Clearly, $f_{\zeta, n}(x)=\mathrm{e}^{-\zeta x} f_{n}(x) / \ell(\zeta)^{n}$ is the Lebesgue density of the $n$-fold convolution of $Q_{\zeta}$. By (A4), the local central limit theorem holds for $f_{\zeta, n}$, i.e. the standardized densities $\hat{f}_{\zeta, n}(x)=n^{1 / 2} \sigma f_{\zeta, n}\left(n^{1 / 2} \sigma x+n m\right)$ satisfy

$$
\lim _{n \rightarrow \infty} \sup _{x \in \mathbb{R}}\left|\hat{f}_{\zeta, n}(x)-(2 \pi)^{-1 / 2} \mathrm{e}^{-x^{2} / 2}\right|=0 .
$$

For any $y$ with $f_{n}(y) \neq 0$, we obtain

$$
\begin{aligned}
\frac{f_{n-1}(y-x)}{f_{n}(y)}= & \frac{\mathrm{e}^{-\zeta x}}{\ell(\zeta)} \frac{f_{\zeta, n-1}(y-x)}{f_{\zeta, n}(y)} \\
= & \frac{\mathrm{e}^{-\zeta x}}{\ell(\zeta)}\left(\frac{n}{n-1}\right)^{1 / 2} \\
& \times \frac{\hat{f}_{\zeta, n-1}\left(\sigma^{-1}\left[(y-n m) /(n-1)^{1 / 2}+(m-x) /(n-1)^{1 / 2}\right]\right)}{\hat{f}_{\zeta, n}\left(\sigma^{-1} n^{1 / 2}[y / n-m]\right)}
\end{aligned}
$$

Hence,

$$
\begin{aligned}
& \left|\frac{f_{n-1}(y-x)}{f_{n}(y)}-\left(\frac{n}{n-1}\right)^{1 / 2} \frac{\mathrm{e}^{-\zeta x}}{\ell(\zeta)}\right| \\
& =\left(\frac{n}{n-1}\right)^{1 / 2} \frac{\mathrm{e}^{-\zeta x}}{\ell(\zeta)} \frac{1}{\hat{f}_{\zeta, n}\left(\sigma^{-1}\left(n^{1 / 2}[y / n-m]\right)\right)} \\
& \quad \times\left|\hat{f}_{\zeta, n-1}\left(\sigma^{-1}\left[\frac{y-n m}{(n-1)^{1 / 2}}+\frac{m-x}{(n-1)^{1 / 2}}\right]\right)-\hat{f}_{\zeta, n}\left(\sigma^{-1} n^{1 / 2}\left[\frac{y}{n}-m\right]\right)\right| .
\end{aligned}
$$

Now let us set $y=s_{n}$. Using the boundedness of $n^{1 / 2}\left[s_{n} / n-m\right]$ and the local central limit theorem for $\hat{f}_{\zeta, n}$, it is easily checked that the difference in absolute value signs tends to 0 uniformly on every compact set of $x$-values, and that $\hat{f}_{\zeta, n}\left(\sigma^{-1} n^{1 / 2}[y / n-m]\right)=$ $\hat{f}_{\zeta, n}\left(\sigma^{-1} n^{1 / 2}\left[s_{n} / n-m\right]\right)$ remains bounded away from 0 , so that its reciprocal remains bounded. This completes the proof.

Corollary 1. Assume that $X_{1}$ has a density $f$ and a finite variance, and that $|\phi|^{n_{0}}$ is Lebesgue integrable for some $n_{0} \in \mathbb{N}$. Then the standard version of $\mathbb{P}_{X_{1} \mid S_{n}=s_{n}}$ converges to $\mathbb{P}_{X_{1}}$ in total variation. If $f$ is bounded (e.g. in the case when $|\phi|$ is Lebesgue integrable), the density of $\mathbb{P}_{X_{1} \mid S_{n}=s_{n}}$ converges uniformly to $f$ on compact sets.

Remark. For well-behaved averages, i.e. $s_{n} / n \rightarrow \mathbb{E}\left[X_{1}\right]$, there is another way of looking at the limiting behavior of $\mathbb{P}_{X_{1} \mid S_{n}=s_{n}}$; only the existence of $\mathbb{E}\left[X_{1}\right]$ has to be assumed. Let $K_{n}(s, \mathrm{~d} x)$ 
be a stochastic kernel which is a regular conditional distribution of $X_{1}$ given that $S_{n}=s$. Let $\mathcal{A}_{n}=\sigma\left(S_{n}, S_{n+1}, S_{n+2}, \ldots\right)$ be the $\sigma$-algebra generated by the tail $\left(S_{n}, S_{n+1}, S_{n+2}, \ldots\right)$ of the sum sequence. Then it is easily checked that $K_{n}\left(S_{n}, \mathrm{~d} x\right)$ is also a regular conditional distribution of $X_{1}$ given $\mathcal{A}_{n}$. The sequence $\mathcal{A}_{n}$ decreases to $\mathcal{A}_{\infty}$, the tail $\sigma$-algebra of $S_{1}, S_{2}, S_{3}, \ldots$ which, by the Hewitt-Savage $0-1$ law, is trivial. Therefore, the martingale convergence theorem yields $\mathbb{E}\left[g\left(X_{1}\right) \mid \mathcal{A}_{n}\right] \rightarrow \mathbb{E}\left[g\left(X_{1}\right)\right]$ almost surely for every bounded measurable function $g: \mathbb{R} \rightarrow \mathbb{R}$, and this implies that

$$
K_{n}\left(S_{n}, B\right) \rightarrow \mathbb{P}_{X_{1}}(B) \quad \text { almost surely for every Borel set } B \subset \mathbb{R} .
$$

In particular, $K_{n}\left(S_{n}, \cdot\right) \rightarrow \mathbb{P}_{X_{1}}$ in distribution almost surely. It follows that the set of sequences $\left(s_{n}\right)_{n \geq 1} \in \mathbb{R}^{\infty}$ for which

$$
\mathbb{P}_{X_{1} \mid S_{n}=s_{n}}=K_{n}\left(s_{n}, \cdot\right) \rightarrow \mathbb{P}_{X_{1}} \quad \text { in distribution }
$$

has probability 1 under the distribution of the full sequence $\left(S_{1}, S_{2}, S_{3}, \ldots\right)$ on the underlying space $\mathbb{R}^{\infty}$. But the set of all sequences $\left(s_{n}\right)_{n \geq 1} \in \mathbb{R}^{\infty}$ satisfying $s_{n} / n \rightarrow \mathbb{E}\left[X_{1}\right]$ also has probability 1 under this distribution (by the law of strong numbers). Therefore, informally stated, given that $S_{n} / n$ takes a value in accordance with the law of large numbers, the conditional distribution of $X_{1}$ is approximately the same as the unconditional distribution of $X_{1}$. Under the conditions of Corollary 1 , it can even been shown that

$$
\lim _{n \rightarrow \infty}\left\|\mathbb{P}_{X_{1} \mid S_{n}}-\mathbb{P}_{X_{1}}\right\| \rightarrow 0 \quad \text { almost surely }
$$

( $\|\cdot\|$ denoting the total variation). It follows that the set of sequences $\left(s_{n}\right)_{n \in \mathbb{N}}$ for which $s_{n} / n \rightarrow \mathbb{E}\left[X_{1}\right]$ and $\left\|\mathbb{P}_{X_{1} \mid S_{n}=s_{n}}-\mathbb{P}_{X_{1}}\right\| \rightarrow 0$ has probability 1 under the distribution of $\left(S_{n}\right)_{n \in \mathbb{N}}$ on the sequence space $\mathbb{R}^{\infty}$.

\section{The case of stable distributions}

It was noted in [15] that, for the (nonintegrable) Cauchy variables, having density $f(x)=$ $\left[\pi\left(1+x^{2}\right)\right]^{-1}$, the density of $\mathbb{P}_{X_{1} \mid S_{n}=n s}$ converges pointwise to $f$ for every $s \in \mathbb{R}$. Loosely speaking, asymptotically, the knowledge of the value of the average does not influence the distribution of $X_{1}$. In this section we determine the asymptotic behavior of $\mathbb{P}_{X_{1} \mid S_{n}=n s}$ for an arbitrary stable distribution for $X_{1}$. For $\alpha \in[0,2]$ and $\beta \in[-1,1]$, let $G_{\alpha, \beta}$ be the stable distribution with characteristic function

$$
\phi_{\alpha, \beta}(u)= \begin{cases}\exp \left\{-|u|^{\alpha} \exp \left(-\frac{\mathrm{i} \pi}{2} \beta K(\alpha) \operatorname{sgn} u\right)\right\}, & \alpha \neq 1, \\ \exp \left\{-|u|\left(\frac{\pi}{2}+\mathrm{i} \beta(\operatorname{sgn} u) \log |u|\right)\right\}, & \alpha=1\end{cases}
$$

where $K(\alpha)=\alpha-1+\operatorname{sgn}(1-\alpha)$. Here we use the parametrization suggested in the monograph [19]. We denote by $g_{\alpha, \beta}(x)$ and $g_{\alpha, \beta}^{(n)}(x \mid s)$ the standard densities of $G_{\alpha, \beta}$ and $\mathbb{P}_{X_{1} \mid S_{n} / n=s}$, respectively.

Theorem 2. For all $s \in \mathbb{R}$,

$$
\lim _{n \rightarrow \infty} g_{\alpha, \beta}^{(n)}(x \mid s)=g_{\alpha, \beta}(x) \quad \text { if }(\alpha, \beta) \in(0,2) \times(-1,1) .
$$


We need several properties of the densities $g_{\alpha, \beta}$ (proofs can be found in [19]).

(i) Smoothness and support. $g_{\alpha, \beta}$ is infinitely differentiable. For $\alpha \in(0,1)$, the support of $G_{\alpha, 1}$ is $\mathbb{R}_{+}$and that of $G_{\alpha,-1}$ is $\mathbb{R}_{-}$; for all other values of $(\alpha, \beta)$, we have $g_{\alpha, \beta}(x)>0$ for all $x \in \mathbb{R}$.

(ii) $L S T$. Let $\ell_{\alpha, \beta}(s)=\int_{\mathbb{R}} \mathrm{e}^{-s x} g_{\alpha, \beta}(x) \mathrm{d} x$. Then $\ell_{\alpha, \beta}(s)<\infty$ in a neighborhood of $s=0$ if and only if $|\beta|=1$ or $\alpha=2$.

(iii) Symmetry. $g_{\alpha, \beta}(-x)=g_{\alpha,-\beta}(x)$.

(iv) Duality. For all $x>0$ and $\alpha \in(1,2]$,

$$
g_{\alpha, \beta}(x)=x^{-1-\alpha} g_{\alpha^{-1}, \beta^{\prime}}\left(x^{-\alpha}\right), \quad \text { where } \quad \beta^{\prime}=1-(2-\alpha)(1+\beta) .
$$

(v) Asymptotics for $\alpha=1$. For all $\beta \in(-1,1]$,

$$
\lim _{x \rightarrow \infty} x^{2} g_{1, \beta}(x)=\frac{1}{2}(1+\beta) .
$$

Proof of Theorem 2. Let $g_{1, \beta}^{* n}$ be the $n$-fold convolution of $g_{\alpha, \beta}$ with itself. To show (1), we need to prove that, for all $\alpha \in(0,2), \beta \in(-1,1)$, and $x, s \in \mathbb{R}$, we have

$$
\lim _{x \rightarrow \infty} \frac{g_{\alpha, \beta}^{*(n-1)}(n s-x)}{g_{\alpha, \beta}^{* n}(n s)}=1 .
$$

By stability, it follows that

$$
\begin{aligned}
\frac{g_{\alpha, \beta}^{*(n-1)}(n s-x)}{g_{\alpha, \beta}^{* n}(n s)} & =\frac{(n-1)^{-1 / \alpha} g_{\alpha, \beta}\left((n-1)^{-1 / \alpha}(n s-x)\right)}{n^{-1 / \alpha} g_{\alpha, \beta}\left(n^{-1 / \alpha} n s\right)} \\
& =\left(\frac{n}{n-1}\right)^{1 / \alpha} \frac{g_{\alpha, \beta}\left((n-1)^{-1 / \alpha}(n s-x)\right)}{g_{\alpha, \beta}\left(n^{1-(1 / \alpha)} s\right)} .
\end{aligned}
$$

By property (i), $g_{\alpha, \beta}$ is continuous at 0 and $g_{\alpha, \beta}(0)>0$.

First let $\alpha \in(0,1)$. Then the arguments of the numerator and denominator of the right-hand side of (3) converge to 0 , and, hence, the ratio tends to 1 .

Now let $\alpha \in(1,2)$. Then, for $s=0$, the right-hand side of (3) tends to 1 as $n \rightarrow \infty$. For $s>0$, the duality (iv) implies that, for all $n$ for which $n s>x$, we have

$$
\begin{aligned}
\frac{g_{\alpha, \beta}^{*(n-1)}(n s-x)}{g_{\alpha, \beta}^{* n}(n s)}= & \left(\frac{n}{n-1}\right)^{1 / \alpha} \\
& \times \frac{\left((n-1)^{1 / \alpha} /(n s-x)\right)^{1+\alpha} g_{\alpha^{-1}, \beta^{\prime}}\left(\left[(n s-x) /(n-1)^{1 / \alpha}\right]^{-\alpha}\right)}{\left(n^{1-1 / \alpha} s\right)^{-(1+\alpha)} g_{\alpha^{-1}, \beta^{\prime}}\left(\left[n^{1-1 / \alpha} s\right]^{-\alpha}\right)},
\end{aligned}
$$

where $\beta^{\prime}=1-(2-\alpha)(1+\beta)$. If $\beta^{\prime} \neq 1$, i.e. $\alpha \neq 2$ and $\beta \neq-1$, the right-hand side of (4) converges to 1 . Next, symmetry property (iii) yields (2) for $\alpha \in(1,2),|\beta|<1$, and $s<0$.

Finally, let $\alpha=1$. By stability,

$$
g_{1, \beta}^{* n}(x)=n^{-1} g_{1, \beta}\left(n^{-1} x-\beta \log n\right), \quad x \in \mathbb{R} .
$$


Together with asymptotic property (v), this yields (2) for $\beta \in(-1,0)$, and the symmetry property gives the same conclusion for $\beta \in(0,1)$. For $\beta=0$, we use the continuity of $g_{1,0}$ and the relation $g_{1,0}(s)>0$ for all $s$ to obtain

$$
\frac{g_{1,0}^{*(n-1)}(n s-x)}{g_{1,0}^{* n}(n s)}=\frac{n}{n-1} \frac{g_{1,0}\left((n-1)^{-1}(n s-x)\right)}{g_{1,0}(s)} \rightarrow 1 \text { as } n \rightarrow \infty .
$$

This completes the proof.

For the extreme stable distributions, i.e. in the case $\beta=1$, the situation is different.

Theorem 3. (a) If $\alpha \in(0,1)$,

$$
\lim _{n \rightarrow \infty} g_{\alpha, 1}^{(n)}(x \mid s)=\exp \left\{\left(\frac{s}{\alpha}\right)^{\alpha /(1-\alpha)}-\left(\frac{s}{\alpha}\right)^{1 /(\alpha-1)} x\right\} g_{\alpha, 1}(x) \text { for all } s>0, x>0 .
$$

(b) If $\alpha=1$,

$$
\lim _{n \rightarrow \infty} g_{1,1}^{(n)}(x \mid s)=\exp \left\{\mathrm{e}^{-s-1}(1+s-x)\right\} g_{1,1}(x) \quad \text { for } s \in \mathbb{R}, x \in \mathbb{R} .
$$

(c) If $\alpha \in(1,2)$,

$$
\lim _{n \rightarrow \infty} g_{\alpha, 1}^{(n)}(x \mid s)= \begin{cases}\exp \left\{-\left(\frac{|s|}{\alpha}\right)^{\alpha /(1-\alpha)}-\left(\frac{|s|}{\alpha}\right)^{1 /(\alpha-1)} x\right\} g_{\alpha, 1}(x) & \text { for } s<0, x \in \mathbb{R}, \\ g_{\alpha, 1}(x) & \text { for } s \geq 0, x \in \mathbb{R} .\end{cases}
$$

Proof. By Theorem 2.6.1 of [19], the LST $\ell_{\alpha, 1}(\zeta)$ of $G_{\alpha, 1}$ is finite for all $\zeta \geq 0$ and given by

$$
\ell_{\alpha, 1}(\zeta)= \begin{cases}\mathrm{e}^{-\zeta^{\alpha}} & \text { if } \alpha \in(0,1) \\ \zeta^{\zeta} & \text { if } \alpha=1 \\ \mathrm{e}^{\zeta^{\alpha}} & \text { if } \alpha \in(1,2]\end{cases}
$$

A short calculation shows that the equation $\ell_{\alpha, 1}^{\prime}(\zeta) / \ell_{\alpha, 1}(\zeta)=-s$ has a unique positive solution $\zeta(s)$ for $s \in(0, \infty)$ if $\alpha \in(0,1)$, for $s \in \mathbb{R}$ if $\alpha=1$, and for $s \in(-\infty, 0)$ if $\alpha \in(1,2)$. The solution is given by

$$
\zeta(s)= \begin{cases}\left(\frac{s}{\alpha}\right)^{1 /(\alpha-1)} & \text { if } \alpha \in(0,1), s>0, \\ \left(\frac{|s|}{\alpha}\right)^{1 /(\alpha-1)} & \text { if } \alpha \in(1,2), s<0 \\ \mathrm{e}^{-s-1} & \text { if } \alpha=1, s \in \mathbb{R} .\end{cases}
$$

Now, from Theorem 1 and (4), where the ratio on the right-hand side also converges to 1 for $\alpha \neq 2, \beta=1$, and $s>0$, we can conclude that

$$
\lim _{n \rightarrow \infty} g_{\alpha, 1}^{(n)}(x \mid s)= \begin{cases}\frac{\mathrm{e}^{-\zeta(s) x}}{\ell_{\alpha, 1}(\zeta(s))} g_{\alpha, 1}(x) & \text { for } \alpha \in(0,1), s \geq 0, x>0, \\ & \text { or } \alpha \in(1,2), x \in \mathbb{R}, s<0, \\ & \text { or } \alpha=1, s \in \mathbb{R}, x \in \mathbb{R}, \\ g_{\alpha, 1}(x) & \text { for } \alpha \in(1,2), x \in \mathbb{R}, s \geq 0 .\end{cases}
$$

The theorem follows by inserting (5) into (6). 


\section{Asymptotic behavior conditional on a fixed sum}

We now consider positive random variables $X_{i}$ with distribution function $F$, LST $\ell$, and a Lebesgue density $f$ on $(0, \infty)$. It is assumed that $f_{n}$, the density of $S_{n}$, is continuous for all $n \geq n_{0}$ and that $\ell$ can be written in the form

$$
\ell(\zeta)=K \zeta^{-\rho} L(\zeta), \quad \zeta \in(0, \infty)
$$

for some constants $K, \rho>0$ and some function $L$ that is slowly varying at $\infty$. By a well-known Tauberian theorem (see [5, Chapter XIII.5]) this implies that

$$
F(x) \sim \frac{K x^{\rho}}{\Gamma(\rho+1)} L\left(\frac{1}{x}\right) \text { as } x \rightarrow 0 .
$$

We impose a somewhat stronger condition on the density $f$ :

- $f$ is continuous on $(0, \eta)$ for some $\eta>0$ and $A:=\lim _{x \rightarrow 0} x^{1-\rho} f(x)$ exists.

Next we introduce $\tilde{\ell}(z)$, the analytic continuation of $\ell$ on the complex half-plane $\operatorname{Re} z>0$, and $\tilde{L}(z)=K^{-1} z^{\rho} \tilde{\ell}(z)$, the analytic continuation of $L$ on $\operatorname{Re} z>0$, where $z^{\rho}=\mathrm{e}^{\rho \log z}$ is the complex power function with $\log z$ denoting the principal branch of the complex logarithm. Our assumption on $\tilde{L}$ is the following.

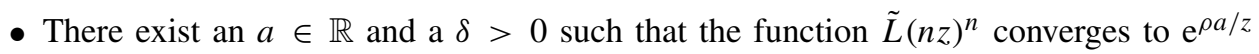
uniformly on every set of $z$-values $z=x+\mathrm{i} y$ with $|y|<\delta$ and $x$ in some compact subset of $(0, \infty)$. Moreover, for every $x>0$, the sequence of functions $y \mapsto \tilde{L}(n(x+\mathrm{i} y))^{n}$ is uniformly bounded on $\mathbb{R}$.

This technical condition is, for example, satisfied for the entire class of distributions that have a rational LST (which contains the phase-type distributions). To see this, note that if $\ell$ is a rational LST, it is of the form

$$
\ell(\zeta)=\sum_{i=0}^{k} a_{i} \zeta^{i} / \sum_{j=0}^{k+l} b_{j} \zeta^{j}=\frac{a_{k}}{b_{k+l}} \zeta^{-l} \frac{\zeta^{k}+\sum_{i=0}^{k-1}\left(a_{i} / a_{k}\right) \zeta^{i}}{\zeta^{k}+\sum_{j=0}^{k+l-1}\left(b_{j} / b_{k+l}\right) \zeta^{j-l}}
$$

for certain constants $k \in \mathbb{Z}_{+}, l \in \mathbb{N}$, and $a_{0}, \ldots, a_{k}, b_{0}, \ldots, b_{k+l} \in \mathbb{R}$, where $a_{k} \neq 0 \neq b_{k+l}$. Hence, we can set $K=a_{k} / b_{k+l}, \rho=l$, and

$$
\tilde{L}(z)=\frac{1+\sum_{i=0}^{k-1}\left(a_{i} / a_{k}\right) z^{i-k}}{1+\sum_{j=0}^{k+l-1}\left(b_{j} / b_{k+l}\right) z^{j-k-l}} .
$$

Now if $\operatorname{Re} z$ remains bounded away from 0 , we find that

$$
(\tilde{L}(n z))^{n}=\left(\frac{1+a_{k-1} / a_{k} z n+O\left(n^{-2}\right)}{1+b_{k+l-1} / b_{k+l} z n+O\left(n^{-2}\right)}\right)^{n} \rightarrow \exp \left\{\left(\frac{a_{k-1}}{a_{k}}-\frac{b_{k+l-1}}{b_{k+l}}\right) \frac{1}{z}\right\} \quad \text { as } n \rightarrow \infty,
$$

and the uniformity and boundedness requirements are clearly satisfied.

Theorem 4. Under the above conditions, the standard version of $\mathbb{P}_{n X_{1} \mid S_{n}=s}$ converges in total variation to the gamma distribution with density $\Gamma(\rho)^{-1}(\rho / s)^{\rho} x^{\rho-1} \mathrm{e}^{-\rho x / s}$. 
Proof. Fix $s>0$, and let $n>n_{0}$. The standard version of $\mathbb{P}_{n X_{1} \mid S_{n}=s}$ has the density

$$
g_{n, s}(x)=\frac{f_{n-1}(s-x / n)}{n f_{n}(s)} f\left(\frac{x}{n}\right) \mathbf{1}_{(0, n s]}(x) .
$$

We define the functions $c(x)$ and $c_{n}(x)$ by

$$
c(x)=\Gamma(\rho) x^{1-\rho} f(x), \quad c_{n}(x)=\Gamma(n \rho) x^{1-n \rho} f_{n}(x) .
$$

Then we can write $g_{n, s}(x)$ in the form

$$
\begin{aligned}
g_{n, s}(x)= & \frac{c_{n-1}(s-x / n) c(x / n)}{c_{n}(s)} \frac{\Gamma(n \rho) n^{-\rho}}{\Gamma((n-1) \rho) \Gamma(\rho)} \\
& \times\left(1-\frac{x}{n s}\right)^{(n-1) \rho-1} s^{-\rho} x^{\rho-1} \mathbf{1}_{(0, n s]}(x) .
\end{aligned}
$$

Clearly, we have, for every $x>0$, by Stirling's formula,

$$
\begin{aligned}
\lim _{n \rightarrow \infty} & \frac{\Gamma(n \rho) n^{-\rho}}{\Gamma((n-1) \rho) \Gamma(\rho)}\left(1-\frac{x}{n s}\right)^{(n-1) \rho-1} s^{-\rho} x^{\rho-1} \mathbf{1}_{(0, n s]}(x) \\
& =\frac{1}{\Gamma(\rho)}\left(\frac{\rho}{s}\right)^{\rho} x^{\rho-1} \mathrm{e}^{-\rho x / s} .
\end{aligned}
$$

Regarding the other factors on the right-hand side of (8) we now show that

$$
\begin{gathered}
\lim _{n \rightarrow \infty} K^{-n} c_{n}(x)=\mathrm{e}^{a x} \quad \text { uniformly on any compact interval in }(0, \infty), \\
\lim _{x \rightarrow 0} c(x)=K .
\end{gathered}
$$

Equations (10)-(11) yield

$$
\lim _{n \rightarrow \infty} \frac{c_{n-1}(s-x / n) c(x / n)}{c_{n}(s)}=1 .
$$

It follows from (8), (9), and (12) that the density of $\mathbb{P}_{n X_{1} \mid S_{n}=s}$ converges pointwise to that of the gamma distribution with shape parameter $\rho$ and scale parameter $\rho / s$. This is sufficient for convergence in total variation.

It remains to prove (10) and (11). Observe first that the constants $K, \rho$, and $A$ are connected by the equation $K=A \Gamma(\rho)$. To see this, note that by the conditions on $f$ we have $F(x) \sim$ $A x^{\rho} / \rho$ as $x \rightarrow 0$, so, by (7),

$$
\frac{A x^{\rho}}{\rho} \sim \frac{K x^{\rho}}{\Gamma(\rho+1)} L\left(\frac{1}{x}\right) \text { as } x \rightarrow 0 .
$$

By our assumption on $\tilde{L}(n z)$, setting $z=1$ we obtain $L(n)^{n} \rightarrow \mathrm{e}^{\rho a}$, so $L(n) \rightarrow 1$. Relation (13) now gives $K=A \Gamma(\rho)$. By the definition of $c(x)$, this implies that $\lim _{x \rightarrow 0} c(x)=$ $\Gamma(\rho) A=K$, i.e. $(11)$.

The remaining proof is based on Laplace inversion, which yields, for arbitrary $c>0$,

$$
f_{n}(y)=\frac{1}{2 \pi \mathrm{i}} \int_{c-\mathrm{i} \infty}^{c+\mathrm{i} \infty} \mathrm{e}^{y z} \tilde{\ell}(z)^{n} \mathrm{~d} z .
$$


Thus,

$$
c_{n}(x)=\frac{\Gamma(n \rho)}{x^{n \rho-1}} \frac{1}{2 \pi} \int_{c-\mathrm{i} \infty}^{c+\mathrm{i} \infty} K^{n} \mathrm{e}^{x z} z^{-n \rho} \tilde{L}(z)^{n} \mathrm{~d} z .
$$

Substituting $z=(n a \rho / s) w$ and setting $c=a n \rho / s$ we obtain

$$
\begin{aligned}
K^{-n} c_{n}(x) & =\frac{\Gamma(n \rho)}{(n \rho)^{n \rho-1}} \frac{1}{2 \pi \mathrm{i}} \int_{1-\mathrm{i} \infty}^{1+\mathrm{i} \infty} \mathrm{e}^{n \rho w} w^{-n \rho} \tilde{L}\left(\frac{n \rho w}{x}\right)^{n} \mathrm{~d} w \\
& =\frac{\Gamma(n \rho)}{(n \rho)^{n \rho-1}} \frac{\mathrm{e}^{n \rho}}{2 \pi} \int_{-\infty}^{\infty} \mathrm{e}^{\mathrm{i} n \rho t}(1+\mathrm{i} t)^{-n \rho} \tilde{L}\left(\frac{n \rho}{x}(1+\mathrm{i} t)\right)^{n} \mathrm{~d} t .
\end{aligned}
$$

We fix a $\varepsilon \in\left(\frac{1}{3}, \frac{1}{2}\right)$, and write the integral on the right-hand side of (14) as $I_{n}^{(1)}+I_{n}^{(2)}$, where $I_{n}^{(1)}$ and $I_{n}^{(2)}$ denote the corresponding integrals over $\left[-n^{-\varepsilon}, n^{-\varepsilon}\right]$ and $\mathbb{R} \backslash\left[-n^{-\varepsilon}, n^{-\varepsilon}\right]$, respectively. By the uniform boundedness condition on $\tilde{L}(n z)^{n}$, there is a constant $C$ such that

$$
\begin{aligned}
\left|I_{n}^{(2)}\right| & \leq \int_{n^{-\varepsilon}}^{\infty} C|1+\mathrm{i} t|^{-n \rho} \mathrm{d} t \\
& =C \int_{n^{-\varepsilon}}^{\infty}\left|1+t^{2}\right|^{-n \rho / 2} \mathrm{~d} t \\
& \leq \frac{C}{n^{-\varepsilon}(n \rho-1)} \\
& =O\left(n^{-1+\varepsilon}\right) \text { as } n \rightarrow \infty .
\end{aligned}
$$

For the second inequality in (15), we have used the estimate (valid for arbitrary $u>0$ )

$$
\begin{aligned}
\int_{u}^{\infty}\left|1+t^{2}\right|^{-n \rho / 2} \mathrm{~d} t & \leq \frac{\left(1+u^{2}\right)^{1 / 2}}{u} \int_{u}^{\infty} \frac{t}{\left(1+t^{2}\right)^{1 / 2}}\left|1+t^{2}\right|^{-n \rho / 2} \mathrm{~d} t \\
& =\frac{\left(1+u^{2}\right)^{1 / 2}}{u} \frac{1}{n \rho-1}\left(1+u^{2}\right)^{-(n \rho-1) / 2} \\
& =\frac{1}{u(n \rho-1)}\left(1+u^{2}\right)^{-n \rho / 2+1} .
\end{aligned}
$$

From (14)-(15) and Stirling's formula, it follows that

$$
\begin{aligned}
K^{-n} c_{n}(x)= & \left(\frac{n \rho}{2 \pi}\right)^{1 / 2} \int_{-n^{-\varepsilon}}^{n^{-\varepsilon}} \mathrm{e}^{\mathrm{i} n \rho t}(1+\mathrm{i} t)^{-n \rho} \tilde{L}\left(\frac{n \rho}{x}(1+\mathrm{i} t)\right)^{n} \mathrm{~d} t+\left(\frac{n \rho}{2 \pi}\right)^{1 / 2} O\left(n^{-1+\varepsilon}\right) \\
= & \int_{\mathbb{R}}(2 \pi)^{-1 / 2} \mathbf{1}_{\left[-\rho^{1 / 2} n^{1 / 2-\varepsilon}, \rho^{1 / 2} n^{1 / 2-\varepsilon}\right]}(u) \mathrm{e}^{\mathrm{i}(n \rho)^{1 / 2} u}\left(1+\mathrm{i} \frac{u}{(n \rho)^{1 / 2}}\right)^{-n \rho} \\
& \times \tilde{L}\left(\frac{n \rho}{x}\left(1+\mathrm{i} u(n \rho)^{-1 / 2}\right)\right)^{n} \mathrm{~d} u+o(1),
\end{aligned}
$$

where we have used $\varepsilon<\frac{1}{2}$ and the substitution $u=(n \rho)^{1 / 2} t$ for the second equality. Consider the integrand on the right-hand side of (16). For the $\tilde{L}$-term, we have, according to our assumptions,

$$
\lim _{n \rightarrow \infty} \sup _{u \in\left[-\rho^{1 / 2} n^{1 / 2-\varepsilon}, \rho^{1 / 2} n^{1 / 2-\varepsilon}\right]}\left|\tilde{L}\left(n\left[\frac{\rho}{x}+\mathrm{i} \frac{\rho}{x} u(n \rho)^{-1 / 2}\right]\right)^{n}-\mathrm{e}^{a x}\right|=0,
$$


and this convergence is uniform in $s$ as long as $s$ is restricted to an arbitrary compact interval in $(0, \infty)$. Moreover, by Taylor's expansion,

$$
\begin{aligned}
& \mathrm{e}^{\mathrm{i}(n \rho)^{1 / 2} u}\left(1+\mathrm{i} \frac{u}{(n \rho)^{1 / 2}}\right)^{-n \rho} \\
& =\exp \left\{\mathrm{i}(n \rho)^{1 / 2} u-n \rho \log \left(1+\frac{\mathrm{i} u}{(n \rho)^{1 / 2}}\right)\right\} \\
& =\exp \left\{\mathrm{i}(n \rho)^{1 / 2} u-n \rho\left(\frac{\mathrm{i} u}{(n \rho)^{1 / 2}}-\frac{1}{2}\left[\frac{\mathrm{i} u}{(n \rho)^{1 / 2}}\right]^{2}+O\left(\left[\frac{u}{n^{1 / 2}}\right]^{3}\right)\right)\right\} \\
& =\exp \left\{-\frac{u^{2}}{2}+O\left(\frac{u^{3}}{n^{1 / 2}}\right)\right\},
\end{aligned}
$$

and the $O\left(u^{3} / n^{1 / 2}\right)$ term tends to 0 uniformly in $|u| \leq \rho^{1 / 2} n^{1 / 2-\varepsilon}$, which is the interval where the expansion is needed (recall that $\varepsilon>\frac{1}{3}$ ). It follows that $\lim _{n \rightarrow \infty} K^{-n} c_{n}(x)=\mathrm{e}^{a x}$ uniformly on any compact subset of $(0, \infty)$, so $(10)$, and, thus, the theorem, is proved.

Examples. (a) Let the $X_{i}$ be uniformly distributed on $(0,1)$. Then $\ell(\zeta)=\zeta^{-1}\left(1-\mathrm{e}^{-\zeta}\right)$, $\rho=A=K=1$, and $\tilde{L}(z)=1-\mathrm{e}^{-z}$. We have $\tilde{L}(n z)^{n} \rightarrow 1$ uniformly on $\operatorname{Re} z \geq \varepsilon$ for every $\varepsilon>0$. All assumptions are satisfied, $a=1$, and $\mathbb{P}_{n X_{1} \mid S_{n}=s}$ converges in total variation to the exponential distribution with mean $s$.

(b) Let $l \in(0,1], \beta>\alpha>0$, and

$$
f(x)=l \frac{1}{\Gamma(\alpha)} x^{\alpha-1} \mathrm{e}^{-x}+(1-l) \frac{1}{\Gamma(\beta)} x^{\beta-1} \mathrm{e}^{-x},
$$

i.e. $f$ is a mixture of two gamma densities (or a pure gamma density if $l=1$ ). Then, as

$$
\ell(\zeta)=l(1+\zeta)^{-\alpha}+(1-l)(1+\zeta)^{-\beta},
$$

we obtain $\rho=\alpha, K=l, A=l / \Gamma(\alpha)$, and

$$
\tilde{L}(z)=\left(\frac{z}{1+z}\right)^{\alpha}\left[1+\frac{1-l}{l}\left(\frac{1}{1+z}\right)^{\beta-\alpha}\right], \quad \operatorname{Re} z>0 .
$$

A short calculation yields

$$
\tilde{L}(n z)^{n} \rightarrow \begin{cases}\mathrm{e}^{-\alpha / z} & \text { if } \beta-\alpha>1, \\ \mathrm{e}^{[(1-l) / l-\alpha] / z} & \text { if } \beta-\alpha=1,\end{cases}
$$

uniformly on $\operatorname{Re} z \geq \varepsilon$ as $n \rightarrow \infty$ for any $\varepsilon>0$. Therefore, we find that, for $\beta \geq \alpha+1$, all assumptions are satisfied and $\mathbb{P}_{n X_{1} \mid S_{n}=s}$ converges in total variation to the gamma distribution with parameters $\alpha / s$ and $\alpha$. It is an open problem whether this also holds in the case $\beta-1<$ $\alpha<\beta$.

\section{Acknowledgements}

The authors would like to thank the referee and the editor for their constructive comments an suggestions. 


\section{References}

[1] Asmussen, S. And Glynn, P. W. (2007). Stochastic Simulation. Algorithms and Analysis. Springer, New York.

[2] Diaconis, P. and Freedman, D. A. (1988). Conditional limit theorems for exponential families and finite versions of de Finetti's theorem. J. Theoret. Prob. 1, 381-410.

[3] Doney, R. A. (1985). Conditional limit theorems for asymptotically stable random walks. Z. Wahrscheinlichkeitsth. 70, 351-360.

[4] Doney, R. A. And O’Brien, G. L. (1992). Thickened renewal processes. Stoch. Process. Appl. 43, 1-8.

[5] Feller, W. (1971). An Introduction to Probability Theory and Its Applications, Vol. II, 2nd edn. John Wiley, New York.

[6] Greenwood, L. and Perkins, E. (1983). A conditioned limit theorem for random walk and Brownian local time on square root boundaries. Ann. Prob. 11, 227-261.

[7] Holst, L. (1979). Two conditional limit theorems with applications. Ann. Statist. 7, 551-557.

[8] Holst, L. (1981). Some conditional limit theorems in exponential families. Ann. Prob. 9, 818-830.

[9] Hooghiemstra, G. and Kennedy, D. P. (1984). Conditioned limit theorems and heavy traffic. Ann. Prob. 12, 631-634.

[10] Janson, S. (2001). Moment convergence in conditional limit theorems. J. Appl. Prob. 38, 421-437.

[11] Martin-Löf, P. (1970). Statistical Models. Handwritten lecture notes for the academic year 1969-1970, compiled by R. Sundberg, Stockholm University.

[12] Poleshchuk, O. M. (1995). A conditional limit theorem for a random walk with zero drift. Russian Math. Surveys 50, 446-448.

[13] Quine, M. P. (1985). Remainder term estimates in a conditional central limit theorem for integer-valued random variables. J. Austral. Math. Soc. Ser. A 39, 75-85.

[14] Schulte-Geers, E. and Stadje, W. (1988). Some results on the joint distribution of the renewal epochs prior to a given time instant. Stoch. Process. Appl. 30, 85-104.

[15] Van Campenhout, J. M. And Cover, T. M. (1981). Maximum entropy and conditional probability. IEEE Trans. Inf. Theory 27, 483-489.

[16] Yang, H. AND Schrage, L. (2009). Conditions that cause risk pooling to increase inventory. Europ. J. Operat. Res. 192, 837-851.

[17] Zabell, S. L. (1974). A limit theorem for conditional expectations with applications to probability theory and statistical mechanics. Doctoral Thesis, Harvard University.

[18] Zabell, S. L. (1993). A limit theorem for expectations conditional on a sum. J. Theoret. Prob. 6, $267-283$.

[19] Zolotarev, V. M. (1986). One-Dimensional Stable Distributions (Trans. Math. Monogr. 86). American Mathematical Society, Providence, RI. 